\title{
Sistema de distribución de medicamentos en dosis unitarias y errores de medicación
}

\author{
System of Distribution of Drugs in Unit Dose and Errors of Medication
}

\author{
Molina-Trinidad EM ${ }^{1}$, Ramos-Garnica $Y^{2}$, Guerrero-Rendón $B^{3}$
}

\begin{abstract}
:
A Drug Distribution System in Unit Dose (DDSUD) was implemented in the services of Isolated, Internal Medicine, Infants, Surgery, Oncology, Nephrology and Intensive Therapies level III of the Niño DIF Hidalgo hospital, applying the hospital pharmacy model, achieving a decrease medication errors and consequently the incorrect use of medications, based on the pharmacotherapeutic profile and preventing errors in the dose distribution system by pharmaceutical services.
\end{abstract}

Keywords:

Drug Distribution System per Unit Dose, medication errors, national model of hospital pharmacy

Resumen:

Se implementó un Sistema de Distribución de Medicamentos en dosis Unitarias (SDMDU) en los servicios de Aislados, Medicina Interna, Lactantes, Cirugía, Oncología, Nefrología y Terapias Intensivas nivel III del hospital Niño DIF Hidalgo, aplicando el modelo de farmacia hospitalaria, logrando disminuir los errores de medicación y en consecuencia el uso incorrecto de los medicamentos, basándose en el perfil farmacoterapéutico y previniendo errores en el sistema de distribución de dosis por parte de los servicios farmacéuticos.

Palabras Clave:

Sistema de Distribución de Medicamentos por dosis Unitarias, errores de medicación, modelo nacional de farmacia hospitalaria.

\section{Introducción}

A nivel mundial los hospitales siguen un sistema de dispensación acorde a las necesidades y políticas de cada unidad. Actualmente en países de América Latina y en Estados Unidos se basa en las normas que marca la OMS y los requerimientos avalan el sistema que sigue la FDA. En nuestro país se siguen las mismas políticas y se diseña e implementar un sistema para la dispensación de medicamentos a los pacientes internos; sin embargo, es común observar que la farmacoterapia no siempre tiene el éxito esperado en tiempo y en efectividad.

El sistema de distribución de medicamentos en dosis unitarias es un sistema diseñado y probado científicamente, que mejora la seguridad de los pacientes y la calidad de la terapéutica en los hospitales.
El proceso de prescripción, validación y administración de medicamentos ha evolucionado y actualmente se considera que los sistemas de prescripción electrónica asistida y el soporte a la toma de decisiones clínica son estrategias prometedoras en la prevención de errores de medicación, por lo que el Servicio de Farmacia, es un objetivo estratégico, que facilita la asistencia farmacéutica al paciente y nos permite mejorar los procesos relacionados con el uso de los medicamentos. El uso de medicamentos hoy en día es de gran utilidad ya que representa una estrategia terapéutica importante. Sin embargo, hay un cierto grado de peligrosidad inherente a su acción farmacológica o a su uso, que ocurre a cada paso del proceso de atención de salud. En conjunto puede conducir al desarrollo de acontecimientos adversos a medicamentos, por ello la seguridad del

\footnotetext{
${ }^{1}$ Autor de correspondencia. Profesora Investigadora. Universidad Autónoma del Estado de Hidalgo. Instituto de Ciencias de la Salud, Pachuca, Hidalgo. México. ORCID: 0000-0003-4251-1469. Email: eva_molina8849@uaeh.edu.mx

${ }^{2}$ Hospital del Niño DIF, Pachuca, Hidalgo. México. Email: sakura_145402@hotmail.com

${ }^{3}$ Hospital del Niño DIF, Pachuca, Hidalgo. México. Email: soyfresa_97_5@yahoo.com.mx
} 
paciente es un principio fundamental de la atención sanitaria.

Por lo anterior, el uso racional de medicamentos contribuye significativamente al bienestar del individuo y de la sociedad.

El error de medicación es aquel daño causado por el fármaco (reacciones adversas y sobredosis) y daños por el uso de medicamentos, incluyendo reducciones de dosis e interrupciones del tratamiento farmacológico. Así mismo, los eventos adversos con medicamentos pueden resultar de errores de medicación.

\section{Historia}

A mediados del siglo pasado se produce el desarrollo de la farmacia hospitalaria, permitiendo al licenciado en Farmacia tener un papel activo en el equipo de salud constituido por los licenciados de Médico Cirujano y Enfermería principalmente además de mantener una participación directa con el paciente. Esta innovación se inicia en los Estados Unidos de Norteamérica en los años de 1950 periodo en el cual se establecieron los principios, conceptos y prácticas que sentarían las bases del progreso de la farmacia hospitalaria. ${ }^{1}$

En la década de los sesentas se pone en práctica el sistema de dispensación de medicamentos programas pilotos de dispensación.

En 1961 Heller establece la terminología de dosis unitarias; y en 1963 Barker publico el trabajo "proyecto de desarrollo del sistema centralizado de dispensación en dosis unitaria". Posteriormente Tester y Parker publicaron los resultados de sus trabajos donde se definieron claramente los objetivos y alcances del sistema de dosis unitaria, existiendo variaciones que hicieron que el sistema se adapte a cada hospital, surgiendo de esta manera el sistema de distribución en dosis unitarias, que representa un significativo cambio en reacción a los sistemas tradicionales de distribución de medicamentos. ${ }^{1}$ En España el sistema de dosis unitarias mantuvo una mejor aceptación gracias a las leyes de seguridad social del país, implementados por primera vez en 1971 en el Nosocomio "Residencia 20 de noviembre" y en 1973 se establece la oficina de farmacia como servicio general clínico del hospital, permitiendo el desarrollo de la farmacia hospitalaria en toda España. ${ }^{1}$

En la década de los ochentas en Estados Unidos aparecieron los sistemas automatizados de almacenamiento y distribución de medicamento con el fin de aumentar la eficiencia del proceso de dispensación, de esta forma se logró disminuir el retraso para cumplir una nueva prescripción médica, así como el exceso de trabajo derivado de los cambios en las prescripción y el desorden que implica que las enfermeras tomen las dosis de otros pacientes o del stock de la sala cuando no se encuentra localizado el medicamento en el cajetín del paciente. En resumen; se incrementa la productividad, la exactitud y el control de la utilización de los medicamentos, todo lo cual mejora la asistencia sanitaria al paciente.

En México en la cultura prehispánica los mexicas tenían un control en la farmacia, ya que estaba institucionalizada y sistematizada por los Panamacani que eran los especialistas dedicados a la preparación y dispensación de remedios y medicamentos.

Con la conquista de la gran Tenochtitlán inicia una nueva faceta en la historia de la farmacia en México, donde los conocimientos mexicas fueron sustituidos por conocimientos adquiridos de Europa; sin embargo, gracias a algunos documentos y códices conservados por Fray Bernardino de Sahagún y por el protomédico Francisco Hernández fue posible salvar una parte de los conocimientos fundamentados en códices prehispánicos de la cultura mexica relacionados con las disciplinas de medicina y farmacia. ${ }^{2}$

En el siglo XV, en la Nueva España se tienen las primeras disposiciones sanitarias, por mencionar el 11 de enero de 1527 , se dispuso imponer multas a quienes curaban sin autorización y qué el 15 de octubre de 1535 , no se ejerciera la profesión de boticario si no se tenían estudios universitarios; por lo cual se creó una institución rectora y supervisora "El Protomedicato" quien examinaba y otorgaba licencias y cédulas para médicos, cirujanos y boticarios.

En 1833 surge el primer código sanitario en el país en el cual establecía que para establecer una botica se debe de contar con un profesional farmacéutico. En 1890 se reforma el código sanitario permitiendo que cualquier ciudadano pueda adquirir una farmacia, proliferando boticas en manos de personas sin la preparación adecuada.

En el primer cuarto siglo XX, creció la tecnología y la ciencia, lo cual dio impulso de manera notable las ciencias médicas y farmacéuticas, específicamente lo referido a la producción masiva de medicamentos. En las últimas décadas del siglo $X X$ la presencia del farmacéutico fue nula, la población podía adquirir medicinas de patente sin acudir al médico y el farmacéutico solo figuraba en establecimientos que distribuían medicamento controlado como responsable sanitario. ${ }^{2}$

\section{Situación actual en México}

Cada centro hospitalario en nuestro país diseña e implementa un sistema para la dispensación de medicamentos a los pacientes internos; sin embargo, es común observar que la farmacoterapia no siempre tiene el éxito esperado en tiempo y en efectividad. Esto se debe en parte, a los errores de medicación que, en algunos países, han sido considerados un problema de salud pública.

La adecuada administración y dispensación de medicamentos tanto en el ámbito hospitalario como en el ambulatorio, contribuyen al buen uso de insumos en beneficio del paciente y del hospital.

Adicionalmente, coadyuva al uso racional de los recursos financieros en este ámbito lo que genera un impacto positivo sobre su gasto total.

El modelo nacional de farmacia hospitalaria, editado en 2009 tiene el objetivo de ser guía para la implementación de los servicios farmacéuticos en México.

En México diferentes estados de la República implementan el sistema de dosis en base a su sistema organizacional y a los requerimientos de medicamentos. 
Por ejemplo, El Hospital Regional de Alta Especialidad de Oaxaca, tiene implementado un servicio de dosis unitaria, el cual ha mejorado en gran medida el sistema de distribución de medicamentos, logrando ahorros económicos y aumentando la seguridad de los pacientes. El Hospital del Instituto Mexicano del Seguro Social No.1 ubicado en Cuernavaca, Morelos, cuenta con un Centro de Información de Medicamentos, el cual ha sido de gran ayuda en la elaboración de criterios para el uso de medicamentos.

El Hospital Universitario de Puebla, destaca su participación dentro del Comité de Farmacia y Terapéutica, y la integración al equipo de salud del profesional farmacéutico, interaccionando con el paciente.

El Hospital del Niño DIF, localizado en Pachuca, Hidalgo, ha logrado la implementación de servicios farmacéuticos hospitalarios en pediatría. Una de las principales fortalezas en la incorporación de farmacéutico en el pase de visita, así como su sistema de distribución por dosis unitarias. Cuenta con Licenciados en Farmacia con gran experiencia en el área, quienes, a partir del 2012, como parte del Programa de Procesos de Calidad y Seguridad del paciente llevan a cabo la notificación sistemática de errores de medicación en los pacientes hospitalizados a los cuales se les suministra medicamentos como parte del Sistema de Dosis Unitarias.

En el Instituto Jalisciense de Alivio al Dolor y Cuidados Paliativos, ubicado en Zapopan, Jalisco, se realizan actividades farmacéuticas en el Centro Integral de Servicios Farmacéuticos ubicado dentro del mismo Instituto, donde ejercen la práctica de la Farmacovigilancia, la Educación Sanitaria y la integración al equipo de salud y cuenta con programas de servicio social, prácticas profesionales y tesis para estudiantes de QFB y Lic. En Farmacia, mediante colaboración con diversas universidades del país.

Actualmente en nuestro país se han realizado mejoras en los sistemas de dispensación de medicamentos considerando el sistema de distribución de medicamentos por dosis unitaria (SDMDU), que consiste en la aplicación de un método de dispensación y control de medicamentos en los servicios de salud, empleado para dispensación y control de los mismos en dosis unitarias a pacientes hospitalizados, cuya organización y coordinación la realizan los licenciados en farmacia. ${ }^{1}$

El sistema de distribución por dosis unitaria es el que mejor ofrece la oportunidad para efectuar un adecuado seguimiento a la terapia con el uso de medicamentos en el paciente. Este sistema permite intervenir en forma oportuna el seguimiento farmacoterapéutico en el paciente antes de la aplicación del medicamento. Se ha demostrado en varios estudios que este sistema es el más seguro para el paciente, es decir, es el más eficiente. $^{2}$

Por otra parte, el profesional farmacéutico lleva acabo actividades asistenciales que son: la selección de medicamento, racionalización de tratamiento, búsqueda de alternativas farmacoterapeuticas, entre otros, por esta razón es importante considerar los conocimentos básicos de farmacoterapia para participar e intervenir adecuadamente en un determinado tratamiento y principalmente el acercamiento en el equipo de salud constituido principalmente por el Médico y los servicios de enfermeria. ${ }^{3}$

\section{Dispensación}

La dispensación de medicamentos es una de las actividades fundamentales del profesionista farmacéutico asociado a la entrega y distribución de los mismos como la validación de prescripcion medica y la información sobre el medicamento, además de sugerir sobre la correcta administración del medicamento y respecto al almacenamiento y preparación de dosis indicadas para el paciente hospitalizado y el ambulatorio. ${ }^{3}$

Por otra parte, el sistema de distribucion de medicamentos por dosis unitarias, cubre las necesidades de medicamentos e insumos del paciente hospitalizado para 24 horas, con el medicamento perfectamente identificado como la establece la norma oficial mexicana NOM-072-SSA1-2012, etiquetado de medicamentos y remedios herbolarios; y el abastecimiento de "stocks" o botiquines especializados de planta. $^{3}$

Para el paciente amibulatorio se dispensa la terapia completa y en caso de tratamientos crónicos, la terapia se realiza durante un mes o el tiempo máximo de prescripción para este tipo de pacientes en el reglamento institucional vigente. ${ }^{3}$

De todos los sistemas de distribucion de medicamentos, el SDMDU ofrece varias ventajas así como la oportunidad para efectuar un adecuado seguimiento a la terapia medicamentosa del paciente; esta acciones del farmacéutico dan una mayor seguridad sobre la administración del medicamentos, reduciendo gastos por consumo de medicamentos innecesarios. ${ }^{3}$

En el hospital de niño DIF de Pachuca, Hidalgo, la implementacion del sistema SDMDU en los servicios clínicos, medicina interna, lactantes, cirugía, oncología, nefrología y terapias intensivas ayudan a un mayor aprovechamiento de los recursos profesionales, ya que siempre demandan un stock de medicamentos.

Además en la central de mezclas endovenosas el profesionista farmacéutico ofrece al paciente hospitalizado la elaboración, acondicionamiento y distribución de mezclas intravenosas asegurando la asepsia, cubriendo las necesidades terapéuticas de medicamentos controlados y otras mezclas medicamentosas.

La preparación de este tipo de mezclas requiere, además de los profesionistas de farmacia capacitados; campana de flujo laminar, area blanca adecuada para este fin, que cumplan con la norma oficial mexicana NOM-249-SSA12010 Mezclas esteriles. $^{3}$

\section{Errores de medicación}

El uso racional de medicamentos contribuye significativamente al bienestar del individuo y por ende, al de la sociedad. Sin embargo esta no es una situación fácil de lograr ni de mantener. La experiencia ha demostrado que en el camino entre la prescripción, la dispensación y la utilización final del medicamento por parte del paciente, a veces surgen problemas que llevan a una incorrecta 
utilización del fármaco o a la presencia de efectos indeseados. ${ }^{4}$

En esta investigación se describen los errores que que pueden ocurrir con la medicación, su prevalencia y las causas de este problema. ${ }^{4}$

A nivel mundial se realizan estudios sobre Acontecimientos Adversos (AA) ocurridos principalmente en pacientes hospitalizados. En los mismos se reportan tasas de incidencia de AA que van desde $2.9 \%$ a $16.6 \%$ del total de admisiones hospitalarias. Del total de AA, $25 \%$ a $50 \%$ se consideraron prevenibles, y entre un $5 \%$ y un $13 \%$ de AA resultaron en la muerte de los pacientes. Dichos estudios sobre incidencia de AA incrementan la necesidad de crear medidas que aumenten la seguridad de los pacientes.

Por otra parte, se define al acontecimiento adverso como el resultado de un daño no intencional o complicación que resulta en un efecto negativo a la salud del paciente: discapacidad al ser dado de alta, o bien, el prolongar los días de estancia hospitalaria o la muerte. El National Coordinating Council for Medication Error Reporting and Prevention (NCCMERP) define los errores de medicación (EM) como: "cualquier incidente prevenible que puede causar daño al paciente o dar lugar a una utilización inapropiada de los medicamentos, cuando éstos están bajo el control de los profesionales sanitarios o del paciente o consumidor. ${ }^{5}$

En los EM se debe analizar desde la perspectiva del sistema, con el fin de documentar diversos aspectos de los mismos. Fundamentalmente conviene clasificarlos en función del proceso de la cadena terapéutica, donde se originan, de la gravedad de las posibles consecuencias para el paciente y de las características del error.

La categoría de gravedad se acompaña de un algoritmo que facilita la correcta clasificación de los errores.

A nivel hospitalario, los procesos en los que se suele observar una frecuencia mas alta de EM son la administración, transcripción y dispensación, aunque muchos de estos errores son triviales y no causan daños en los pacientes. ${ }^{2}$

\section{Recomendaciones sobre dispensación y administración}

La FIP (Féderation Internationale Pharmaceutique) indica a los farmacéuticos y otros profesionales de la salud a educar sanitariamente a los pacientes y a las personas que los atienden, con el fin de aumentar la comprensión del uso adecuado de los medicamentos y de todo lo relacionado con su administración y estimula a los farmacéuticos y otros profesionales sanitarios a participar regularmente en programas prácticos de prevención de errores de medicación, y cuando éstos se presenten a participar activamente en la investigación. La FIP hace las siguientes recomendaciones a los farmacéuticos, diseñadas para reducir errores debidos al etiquetado y envasado de medicamentos y otros productos sanitarios: 1. Los farmacéuticos deben utilizar únicamente medicamentos adecuadamente etiquetados y almacenados. Las etiquetas se deben leer varias veces durante el proceso de dispensación o bien se debe utilizar tecnología informática para controlar la selección. ${ }^{2}$
2. Los farmacéuticos y otros profesionales de la salud que participan en la administración de fármacos deben leer la etiqueta al seleccionar o preparar el medicamento, inmediatamente antes de administrar el mismo y cuando lo devuelvan a su lugar habitual de almacenamiento.

3. Los farmacéuticos y otros profesionales sanitarios deben notificar, en forma confidencial, a través de los programas de notificación adecuados, los errores de medicación ocurridos, cuyos detalles deben quedar a disposición de todos los profesionales de la atención a la salud con el fin de procurar mejoras en el proceso. ${ }^{2}$

4. Los farmacéuticos deben asegurarse de que el paciente, o la persona que cuida al paciente, entienda cómo debe utilizar el medicamento con el fin de conseguir el máximo beneficio terapéutico y evitar efectos adversos o errores en el uso de los medicamentos. Preferiblemente, esto debe reforzarse con una etiqueta que se colocará en los servicios farmacéuticos.

5. Cuando los sistemas nacionales requieran el reenvasado de determinados medicamentos, se deben diseñar políticas y procedimientos para minimizar los errores y se deben seguir la mayoría de recomendaciones relacionadas con el etiquetado. ${ }^{2}$

Los errores de medicación se clasifican en distintos tipos según la naturaleza de los mismos. En 1993 la American Society of Health-System Pharmacists (ASHP) publicó las Directrices para la prevención de errores de medicación en los hospitales, en las que incluyó una clasificación de los EM en 11 tipos. $^{6}$

En la tabla 1 se indican una clasificación de los errores de medicación.



Tablal. Categorías de gravedad los errores de medicación. Adaptación española de la clasificación del NCCERP. ${ }^{6}$

Los EM se clasifican en 13 tipos y varios subtipos, incluyendo algunos no contemplados en la clasificación de la ASHP, por su parte en España realizo una adaptación de la NCCMERP obteniendo una taxonomía que considera 15 tipos de EM. ${ }^{6}$

En la siguiente tabla se mencionan los errores de medicación. 


\section{Agradecimientos}



Tabla 2. Tipos de errores de medicación. Adaptación española de la clasificación del NCCERP. ${ }^{6}$

En la revisión de la literatura se encuentra que la población pediátrica tiene mayor riesgo de sufrir un evento adverso, Moscoso en su investigación "Reacciones Adversas a Medicamentos en el Hospital de Suba Bogotá" identifico que gestantes, niños, adolescentes y adultos jóvenes son los principales grupos de riesgo para presentar un EM (50\%). ${ }^{7}$

\section{Conclusiones}

El establecimiento del Sistema de Distribución de Medicamentos en Dosis Unitarias disminuye los errores de medicación que se presentan en los sistemas tradicionales.

La implementación de este sistema en el Hospital del Niño DIF, ha permitido disminuir los costos por medicamentos, una buena gestión en la selección y adquisición de medicamentos, además de la seguridad para el paciente, permitiendo mejor utilización del tiempo para cada uno de los profesionales del equipo de salud, disminuyendo los errores de medicación en el área de servicios farmacéuticos, así como el inadecuado uso de los medicamentos.
A la Universidad Autónoma del Estado de Hidalgo y al Hospital del Niño DIF de Pachuca, Hidalgo por la asistencia al programa de servicio social que realizan los alumnos de la Licenciatura de Farmacia.

\section{Referencias}

1. Álvarez FKR. Análisis Del Funcionamiento del Sistema de distribución de medicamentos por dosis unitarias en el Centro Médico Naval. Periodo marzo 2003- marzo 2004. Universidad Naval Mayor de San Francisco Fundación en 1551. (Citado junio 2015). Disponible en: http://Cybertesis.unmsm.edu.pe

2. Girón AN. Sistema de distribución de medicamentos por dosis unitarias. Consultora de servicios farmacéuticos. Honduras OPS/ OMS Octubre 1997. (Citado julio 2015). Disponible en: http://sdmdu.pdf

3. Implementación del sistema de distribución de medicamentos en dosis unitaria. Revista colombiana de Ciencias Químico- Farmacéuticas. Disponible en: www.ciencias.unal.edu.com

4. Díaz JA. "Planificación y Organización de los Servicios de Farmacia Hospitalaria", Universidad Nacional de Colombia, Santafé de Bogotá, 1994.

5. García BM. La Alianza Mundial para la Seguridad del Paciente. Directora de la Oficina de Servicios Integrados de Salud. Unidad de Políticas, Sistemas y Servicios Sanitarios. Organización Mundial de la Salud, Oficina Europea. Barcelona (España). (Citado agosto 2018). https://www.pinterest.com.mx/pin/21603273190185410/

6. National Coordinating Council for Medication Error Reporting and Prevention. Taxonomy of medication errors, 1998. Organismo $\begin{array}{llll}\text { Americano. } & \text { (Citado }\end{array}$ www.nccmerp.org/aboutmederrors.htm

7. Moscoso VSM, Ramírez CGF, López GJJ, Gerena UBE. Reacciones adversas a medicamentos en el Hospital de Suba de Bogotá. J Public Health 2006; 8(2): 209-17. 